IP-ASTP-11-94

June, 1994

\title{
Nonfactorizable Contributions to Nonleptonic Weak Decays of Heavy Mesons
}

\author{
Hai-Yang Cheng \\ Institute of Physics, Academia Sinica \\ Taipei, Taiwan 11529, Republic of China
}

\begin{abstract}
Nonfactorizable contributions to exclusive two-body nonleptonic weak decays of heavy mesons arising from color octet currents, characterized by the parameter $r_{2}$, are extracted from the data. It is found that $r_{2}$ is equal to $\sim-0.67,-(0.9 \sim 1.1),-(1.2 \sim 1.3)$, $\sim 0.36$ respectively for $D \rightarrow \bar{K} \pi, \bar{K}^{*} \pi, \bar{K}^{*} \rho, \bar{B} \rightarrow D \pi$ decays. As expected, soft-gluon effects become stronger when the decay particles are less energetic, allowing more time for significant final-state strong interactions. As a consequence, the parameter $a_{2}$ is not universal and is channel or class dependent, contrary to the anticipation of the factorization approach. The leading $1 / N_{c}$ expansion works most successfully for $D \rightarrow \bar{K} \pi$ decays as the subleading $1 / N_{c}$ factorizable contribution is almost compensated by the soft-gluon effect. We argue that, in contrast to what happens in $B^{-} \rightarrow D^{(*)} \pi(\rho)$ decays, the nonfactorizable term and the subleading $1 / N_{c}$ factorizable term in $\bar{B} \rightarrow \psi \bar{K}^{(*)}$ decays are opposite in signs, in accordance with a recent QCD sum rule calculation. Implications are discussed.
\end{abstract}


1. Introduction A common wisdom for having an approximate description of the underlying physics of nonperturbative QCD is to search for the small parameters in the theory, then set the small parameters to zero and treat the finite effects of the parameters as small perturbations $[1,2]$. Hence, even for a nonperturbative physics such as QCD, one can still perform a sensible perturbative calculation. The well-known examples are chiral perturbation theory and the heavy quark effective theory which respresent a good approximation of QCD in the light and heavy quark mass limits, respectively. The corresponding small expansion parameters in these theories are $m_{q} / \Lambda_{\mathrm{QCD}}$ and $\Lambda_{\mathrm{QCD}} / m_{Q}$ with $m_{q}\left(m_{Q}\right)$ being the light (heavy) quark mass.

By treating the number of color degrees of freedom as a free parameter $N_{c}, 1 / N_{c}$ can be another useful small parameter in QCD. The fact that $1 / N_{c}$ is indepedent of energy has its advantage and disadvantage. On the one hand, it can be used as an expansion parameter for both short- and long-distance dynamics owing to its energy independence. On the other hand, whether or not the $1 / N_{c}$ approach works is at best case by case dependent, since, unlike chiral perturbation theory or the heavy quark effective theory, there is no certain kinematic region where the validity of the $1 / N_{c}$ expansion is guaranteed. Empirically, while the large- $N_{c}$ approach fails in some cases, it explains well the OZI rule and even provides quantitative predictions on the relative strength of the baryon-meson coupling constants and on the baryon mass relations up to $\mathcal{O}\left(1 / N_{c}^{2}\right)$, as was realized last year [3]. It is also known for sometime that the leading $1 / N_{c}$ expansion operates reasonably well for exclusive two-body nonleptonic decays of charmed mesons [4]; 1 the discrepancy between theory and experiment for color-suppressed channels e.g. $D^{0} \rightarrow \bar{K}^{0} \pi^{0}$ is greatly improved provided that contributions from Fierz-transformed currents, which are suppressed by order $1 / N_{c}$, are dropped [6,7]. Due to the success of the $1 / N_{c}$ approach to charmed meson decays, it has been widely believed by many practitioners in this field that it applies equally well to the weak decays of bottom mesons.

The recent CLEO data on the $B$ decays $\bar{B} \rightarrow D \pi, D \rho, D^{*} \pi, D^{*} \rho$ exhibit a quite striking result [8]: The interference between the two different amplitudes contributing to exclusive two-body $B^{-}$decays are evidently constructive, contrary to what naively expected from the leading $1 / N_{c}$ expansion. In other words, the observed destructive interference pattern

\footnotetext{
${ }^{1}$ For a review of the $1 / N_{c}$ expansion for nonleptonic weak decays of mesons, see Ref.[5].
} 
in $D^{+} \rightarrow \bar{K}^{0} \pi^{+}, \bar{K}^{0} \rho^{+}, \bar{K}^{* 0} \pi^{+}$cannot be extrapolated to $B$ decays. Though, as stressed before, the rule of retaining only the leading term in the $1 / N_{c}$ expansion is case by case dependent, an understanding of why it is operative for charm decays but not for the $B$ meson case is called for.

Since $N_{c}=3$ in reality, when the $1 / N_{c}$ approach works empirically, it will imply either that the effective expansion parameter is something like e $1 /\left(4 \pi N_{c}\right), 1 / N_{c}^{2} \ldots$, or that there is a dynamic reason for the suppression of non-leading $1 / N_{c}$ terms. In the large- $N_{c}$ limit, the meson decay amplitude is factorizable [4] (this is no longer true for baryon decay as the baryon contains $N_{c}$ quarks.) Irrespective of the $1 / N_{c}$ expansion, the factorization hypothesis means that the meson two-body decay amplitude may be expressed as the product of two independent hadronic currents. Consider the operator

$$
O_{1}=(\bar{s} c)(\bar{u} d)
$$

where $\left(\bar{q}_{1} q_{2}\right)=\bar{q}_{1} \gamma_{\mu}\left(1-\gamma_{5}\right) q_{2}$. The decay amplitude of $D^{+} \rightarrow \bar{K}^{0} \pi^{+}$induced by $O_{1}$ in general can be expressed in terms of factorizable and nonfactorizable contributions:

$$
\left\langle\bar{K}^{0} \pi^{+}\left|O_{1}\right| D^{+}\right\rangle=\left\langle\pi^{+}|(\bar{u} d)| 0\right\rangle\left\langle\bar{K}^{0}|(\bar{s} c)| D^{+}\right\rangle+\left\langle\bar{K}^{0} \pi^{+}\left|O_{1}\right| D^{+}\right\rangle_{n f},
$$

where the subscript $n f$ denotes a nonfactorizable contribution. In the $1 / N_{c}$ expansion, the factorizable amplitude is of order $N_{c}^{1 / 2}$, while the nonfactorizable one is of order $N_{c}^{-1 / 2}$. Using the identity

$$
O_{1}=\frac{1}{N_{c}} O_{2}+\frac{1}{2}\left(\bar{s} \lambda^{a} d\right)\left(\bar{u} \lambda^{a} c\right)
$$

with $O_{2}=(\bar{s} d)(\bar{u} c)$ and $\left(\bar{q}_{1} \lambda^{a} q_{2}\right)=\bar{q}_{1} \gamma_{\mu}\left(1-\gamma_{5}\right) \lambda^{a} q_{2}$, one obtains

$$
\begin{aligned}
\left\langle\bar{K}^{0} \pi^{+}\left|O_{1}\right| D^{+}\right\rangle_{n f} & =\frac{1}{N_{c}}\left\langle\bar{K}^{0} \pi^{+}\left|O_{2}\right| D^{+}\right\rangle_{f}+\frac{1}{2}\left\langle\bar{K}^{0} \pi^{+}\left|\left(\bar{s} \lambda^{a} d\right)\left(\bar{u} \lambda^{a} c\right)\right| D^{+}\right\rangle \\
& +\frac{1}{N_{c}}\left\langle\bar{K}^{0} \pi^{+}\left|O_{2}\right| D^{+}\right\rangle_{n f},
\end{aligned}
$$

where the factorizable amplitude $\left\langle\bar{K}^{0} \pi^{+}\left|O_{2}\right| D^{+}\right\rangle_{f}$ is equal to $\left\langle\bar{K}^{0}|(\bar{s} d)| 0\right\rangle\left\langle\pi^{+}|(\bar{u} c)| D^{+}\right\rangle$. In the traditional vacuum insertion method only the factorizable terms are retained so that

$$
\left\langle\bar{K}^{0} \pi^{+}\left|O_{1}\right| D^{+}\right\rangle_{n f}=\frac{1}{N_{c}}\left\langle\bar{K}^{0} \pi^{+}\left|O_{1}\right| D^{+}\right\rangle_{f}
$$

\footnotetext{
${ }^{2}$ For example, the expansion parameter in QED is $\alpha=e^{2} / 4 \pi$ even though $e \sim \frac{1}{3}$ is not very small.
} 
However, this naive method encounters two problems. First, it is not logically consistent [9]: A nonfactorizable term becomes a subleading $1 / N_{c}$ factorizable contribution. Second, naive factorization cannot explain the bulk of the experimental data of charm decay (for a review, see Ref.[5]). Both problems hint that it is indispensible to take into account nonfactorizable effects. To the order $\mathcal{O}\left(N_{c}^{-1 / 2}\right)$, we thus keep the second nonfactorizable term on the r.h.s. of Eq.(4) and drop the third term as it is further suppressed by a factor of $1 / N_{c}$. To the end, we have

$$
\left\langle\bar{K}^{0} \pi^{+}\left|O_{1}\right| D^{+}\right\rangle=\left\langle\bar{K}^{0} \pi^{+}\left|O_{1}\right| D^{+}\right\rangle_{f}+\left\langle\bar{K}^{0} \pi^{+}\left|O_{2}\right| D^{+}\right\rangle_{f}\left(\frac{1}{N_{c}}+\frac{r_{2}}{2}\right),
$$

where

$$
r_{2}=\frac{\left\langle\bar{K}^{0} \pi^{+}\left|\left(\bar{s} \lambda^{a} d\right)\left(\bar{u} \lambda^{a} c\right)\right| D^{+}\right\rangle}{\left\langle\bar{K}^{0} \pi^{+}|(\bar{s} d)(\bar{u} c)| D^{+}\right\rangle_{f}}
$$

first introduced in Ref.[10], denotes the nonperturbative effects arising from the soft gluon exchange between the color octet currents $\left(\bar{s} \lambda^{a} d\right)$ and $\left(\bar{u} \lambda^{a} c\right)$ relative to that from the corresponding color singlet currents and is of order $1 / N_{c}$ in the large- $N_{c}$ limit.

It is clear from Eq.(6) that if the leading $1 / N_{c}$ expansion works, it will come from the dynamical reason that the subleading $1 / N_{c}$ factorizable contribution is compensated by the nonperturbative correction, namely $r \approx-2 / N_{c}$. Though in practice it is very difficult to estimate the nonperturbative soft gluon effects (for some recent attempts; see Refs.[11-13]), they can be extracted from the available data. Consider the decays $D \rightarrow P P, V P, V V$ and $B \rightarrow P P(P$ : pseudoscalar meson, $V$ : vector meson). It is expected that the soft-gluon effect is such that

$$
\left|r_{2}(D \rightarrow V V)\right|>\left|r_{2}(D \rightarrow V P)\right|>\left|r_{2}(D \rightarrow P P)\right|>\left|r_{2}(B \rightarrow P P)\right|
$$

as the final-state particles have more time to allow significant final-state strong interactions when they become less energetic. The purpose of this paper is to determine the parameter $r_{2}$ from data and confirm the above expectation. Implications on the factorization method are discussed.

2. Charmed Meson Decays In the large- $N_{c}$ approach both soft-gluon nonperturbative effects and final-state interactions are subleading $1 / N_{c}$ nonfactorizable corrections [4]. In 
order to determine the soft-gluon effects, we will focus on the exotic channels e.g. $D^{+} \rightarrow$ $\bar{K}^{0} \pi^{+}, \pi^{0} \pi^{+}$and the decay modes with one single isospin component, e.g. $D^{+} \rightarrow \pi^{+} \phi, D_{s}^{+} \rightarrow$ $\pi^{+} \phi$, where final-state interactions are presumably negligible.

The QCD-corrected weak Hamiltonian for Cabibbo-allowed charm decays is given by

$$
\mathcal{H}_{\mathrm{eff}}=\frac{G_{F}}{\sqrt{2}} V_{u d}^{*} V_{c s}\left(c_{1} O_{1}+c_{2} O_{2}\right),
$$

with $O_{1}=(\bar{s} c)(\bar{u} d), O_{2}=(\bar{s} d)(\bar{u} c), c_{1}$ and $c_{2}$ are Wilson coefficient functions determined at the renormalization scale $\mu \sim m_{c}$

$$
c_{1}\left(m_{c}\right) \sim 1.26, \quad c_{2}\left(m_{c}\right) \sim-0.51
$$

The amplitude for the decay $D^{+} \rightarrow \bar{K}^{0} \pi^{+}$up to the subleading order in $1 / N_{c}$ is

$$
\begin{aligned}
& A\left(D^{+} \rightarrow \bar{K}^{0} \pi^{+}\right)=\frac{G_{F}}{\sqrt{2}} V_{u d}^{*} V_{c s}(a+b), \\
& a=a_{1}\left(m_{D}^{2}-m_{K}^{2}\right) f_{\pi} f_{+}^{D K}\left(m_{\pi}^{2}\right), \\
& b=a_{2}\left(m_{D}^{2}-m_{\pi}^{2}\right) f_{K} f_{+}^{D \pi}\left(m_{K}^{2}\right),
\end{aligned}
$$

where $f_{\pi}=132 \mathrm{MeV}, f_{K}=160 \mathrm{MeV}$,

$$
a_{1}=c_{1}+\xi_{1} c_{2}, \quad a_{2}=c_{2}+\xi_{2} c_{1}
$$

with $\xi_{1}=1 / N_{c}+r_{1} / 2, \xi_{2}=1 / N_{c}+r_{2} / 2$, and

$$
\begin{aligned}
& r_{1}(D \rightarrow \bar{K} \pi)=\frac{\left\langle\pi^{+} \bar{K}^{0}\left|\left(\bar{u} \lambda^{a} d\right)\left(\bar{s} \lambda^{a} c\right)\right| D^{+}\right\rangle}{\left\langle\pi^{+} \bar{K}^{0}|(\bar{u} d)(\bar{s} c)| D^{+}\right\rangle_{f}}=\frac{\left\langle\pi^{+} K^{-}\left|\left(\bar{u} \lambda^{a} d\right)\left(\bar{s} \lambda^{a} c\right)\right| D^{0}\right\rangle}{\left\langle\pi^{+} K^{-}|(\bar{u} d)(\bar{s} c)| D^{0}\right\rangle_{f}} \\
& r_{2}(D \rightarrow \bar{K} \pi)=\frac{\left\langle\pi^{+} \bar{K}^{0}\left|\left(\bar{s} \lambda^{a} d\right)\left(\bar{u} \lambda^{a} c\right)\right| D^{+}\right\rangle}{\left\langle\pi^{+} \bar{K}^{0}|(\bar{s} d)(\bar{u} c)| D^{+}\right\rangle_{f}}=\frac{\left\langle\pi^{0} \bar{K}^{0}\left|\left(\bar{s} \lambda^{a} d\right)\left(\bar{u} \lambda^{a} c\right)\right| D^{0}\right\rangle}{\left\langle\pi^{0} \bar{K}^{0}|(\bar{s} d)(\bar{u} c)| D^{0}\right\rangle_{f}}
\end{aligned}
$$

where we have applied Eq.(6) to derive (11) and isospin symmetry to relate the $D^{+} \rightarrow \bar{K}^{0} \pi^{+}$ matrix elements of color octet currents to that of $D^{0} \rightarrow K^{-} \pi^{+}, \bar{K}^{0} \pi^{0}$. In the literature, factorization often means that the parameters $a_{1}$ and $a_{2}$ (and hence $\xi_{1}$ and $\xi_{2}$ ) are universal, f namely they are channel independent in $D$ or $B$ decays. However, we see from Eq.(13) that a priori there is no reason to expect that $r_{1}$ and $r_{2}$ are decay mode independent.

For the form factor $f_{+}^{D K}\left(q^{2}\right)$ in Eq.(11) we will use the average value

$$
f_{+}^{D K}(0)=0.76 \pm 0.02
$$

\footnotetext{
${ }^{3}$ Note that $\xi_{1}=\xi_{2}=1 / N_{c}$ corresponds to naive factorization, while leading $1 / N_{c}$ expansion leads to $\xi_{1}=\xi_{2}=0$.
} 
extracted from the recent measurements of $D \rightarrow K \ell \bar{\nu}$ by CLEO II, E687 and E691 [14]. As for the form factor $f_{+}^{D \pi}$, there are only two available experimental information. An earlier measurement of the Cabibbo-suppressed decay $D^{0} \rightarrow \pi^{-} \ell^{+} \nu$ by Mark III yields $\left|f_{+}^{D \pi}(0) / f_{+}^{D K}(0)\right|=1.0_{-0.3}^{+0.6} \pm 0.1[14,15]$, while a very recent CLEO-II measurement of $D^{+} \rightarrow \pi^{0} \ell^{+} \nu$ gives $\left|f_{+}^{D \pi}(0) / f_{+}^{D K}(0)\right|=1.29 \pm 0.21 \pm 0.11$ [16]. Though the latter perfers a larger $f_{+}^{D \pi}(0)$ over $f_{+}^{D K}(0)$, its error is still very large. Fortunately we can use the recent measurement of $D^{+} \rightarrow \pi^{+} \pi^{0}$ by CLEO II [17] to fix $f_{+}^{D \pi}(0)$. The amplitude of $D^{+} \rightarrow \pi^{+} \pi^{0}$ reads

$$
A\left(D^{+} \rightarrow \pi^{+} \pi^{0}\right)=\frac{G_{F}}{2 \sqrt{2}} V_{u d}^{*} V_{c s}\left(a_{1}+a_{2}\right)\left(m_{D}^{2}-m_{\pi}^{2}\right) f_{\pi} f_{+}^{D \pi}\left(m_{\pi}^{2}\right),
$$

where $a_{1}$ and $a_{2}$ are defined in the same manner as in (12) except that in the present case $r_{1}(D \rightarrow \pi \pi)=r_{2}(D \rightarrow \pi \pi)$. Assuming a monopole behavior for the form factor

$$
f_{+}\left(q^{2}\right)=\frac{f_{+}(0)}{1-\left(q^{2} / m_{*}^{2}\right)},
$$

where $m_{*}$ is the mass of the low-lying $1^{-}$resonance that couples to the weak current, and using the experimental value [17]

$$
\mathcal{B}\left(D^{+} \rightarrow \pi^{+} \pi^{0}\right)=(0.22 \pm 0.05 \pm 0.05) \%
$$

as well as $\tau\left(D^{+}\right)=10.66 \times 10^{-13} s[18]$, we find [19]

$$
[1+\xi(D \rightarrow \pi \pi)] f_{+}^{D \pi}(0) \approx 0.83
$$

To proceed further we assume that $\xi_{1}(D \rightarrow \bar{K} \pi)=\xi_{2}(D \rightarrow \bar{K} \pi)$ (which is at least valid in the $\mathrm{SU}(3)$ limit $)=\xi(D \rightarrow \pi \pi)$. Substituting (14), (16), (18) into (15) we find $\xi(D \rightarrow \bar{K} \pi) \approx$ $7 \times 10^{-3}$, and hence

$$
r(D \rightarrow \bar{K} \pi) \approx-\frac{2}{3} .
$$

Evidently, the subleading $1 / N_{c}$ factorizable contribution is almost compensated by the nonfactorizable soft gluon effect, so that $\xi \approx 0$. This explains why the leading $1 / N_{c}$ expansion operates for $D \rightarrow \bar{K} \pi$ decays.

We digress for a moment to make a remark on the $W$-exchange amplitude in the decays $D^{0} \rightarrow K^{-} \pi^{+}, \bar{K}^{0} \pi^{0}$, which is given by

$$
\begin{aligned}
A(W-\text { exchange }) & =\frac{G_{F}}{\sqrt{2}} V_{u d}^{*} V_{c s}\left\{c_{2}\left\langle K^{-} \pi^{+}|(\bar{s} d)| 0\right\rangle\left\langle 0|(\bar{u} c)| D^{0}\right\rangle\right. \\
& \left.+c_{1}\left\langle K^{-} \pi^{+}\left|\left(\bar{s} \lambda^{a} d\right)\left(\bar{u} \lambda^{a} c\right)\right| D^{0}\right\rangle\right\} .
\end{aligned}
$$


We see that although the first term vanishes in the SU(3) limit owing to the conservation of vector current, the effect of soft-gluon exchange could be important. Indeed, a general phenomenological analysis of $D \rightarrow \bar{K} \pi$ data indicates that $W$-exchange is small compared to external and internal $W$-emission amplitudes but it is not negligible [20].

We next determine the parameters $r_{1}$ and $r_{2}$ for $D \rightarrow V P$ decays, whose amplitudes are of the form

$$
A(D \rightarrow V P)=M\left(\varepsilon \cdot p_{D}\right)
$$

where $\varepsilon_{\mu}$ is the polarization vector of the vector meson $V$, and $p_{D}$ is the momentum of the charmed meson. We first consider the decay $D^{+} \rightarrow \phi \pi^{+}$which proceeds solely through internal $W$ emission:

$$
\begin{aligned}
A\left(D^{+} \rightarrow \phi \pi^{+}\right) & =\frac{G_{F}}{\sqrt{2}} V_{u s}^{*} V_{c s} a_{2}\langle\phi|(\bar{s} s)| 0\rangle\left\langle\pi^{+}|(\bar{u} c)| D^{+}\right\rangle \\
& =\frac{G_{F}}{\sqrt{2}} V_{u s}^{*} V_{c s} b^{\prime}\left(\varepsilon \cdot p_{D}\right),
\end{aligned}
$$

with

$$
b^{\prime}=2 a_{2} f_{\phi} m_{\phi} f_{+}^{D \pi}\left(m_{\phi}^{2}\right)
$$

where we have applied the relation $\langle\phi|(\bar{s} s)| 0\rangle=f_{\phi} m_{\phi} \varepsilon_{\mu}$. From the measured branching ratio $\mathcal{B}\left(D^{+} \rightarrow \phi \pi^{+}\right)=(6.0 \pm 0.8) \times 10^{-3}[18]$, and the decay rate formula

$$
\Gamma\left(D^{+} \rightarrow \phi \pi^{+}\right)=\left|\frac{G_{F}}{\sqrt{2}} V_{u s}^{*} V_{c s} b^{\prime}\right|^{2}\left(\frac{\left(m_{D}^{2}-m_{\phi}^{2}-m_{\pi}^{2}\right)^{2}}{4 m_{\phi}^{2}}-m_{\pi}^{2}\right),
$$

we obtain

$$
\left|b^{\prime}\left(D^{+} \rightarrow \phi \pi^{+}\right)\right| \approx 0.336 \mathrm{GeV}^{3}
$$

Comparing (25) with (23) leads to $\left(f_{\phi}=0.221\right)$

$$
\xi_{2}\left(D^{+} \rightarrow \phi \pi^{+}\right) \approx-0.12, \quad r_{2}\left(D^{+} \rightarrow \phi \pi^{+}\right) \approx-0.92
$$

It is evident that the soft-gluon effect is larger than that in $D \rightarrow P P$ decay owing to the small relative momentum between the $V P$ final states. 
The decay $D^{+} \rightarrow \bar{K}^{* 0} \pi^{+}$receives both external and internal $W$-emission contributions:

$$
\begin{aligned}
A\left(D^{+} \rightarrow \bar{K}^{* 0} \pi^{+}\right) & =\frac{G_{F}}{\sqrt{2}} V_{u d}^{*} V_{c s}\left(a_{1}\left\langle\pi^{+}|(\bar{u} d)| 0\right\rangle\left\langle\bar{K}^{* 0}|(\bar{s} c)| D^{+}\right\rangle+a_{2}\left\langle\bar{K}^{* 0}|(\bar{s} d)| 0\right\rangle\left\langle\pi^{+}|(\bar{u} c)| D^{+}\right\rangle\right) \\
& =\frac{G_{F}}{\sqrt{2}} V_{u d}^{*} V_{c s}\left(a^{\prime}+b^{\prime}\right)\left(\varepsilon \cdot p_{D}\right)
\end{aligned}
$$

with

$$
\begin{aligned}
& a^{\prime}=a_{1} f_{\pi}\left[\left(m_{D}+m_{K^{*}}\right) A_{1}^{D K^{*}}\left(m_{\pi}^{2}\right)-\left(m_{D}-m_{K^{*}}\right) A_{2}^{D K^{*}}\left(m_{\pi}^{2}\right)\right], \\
& b^{\prime}=2 a_{2} f_{K^{*}} m_{K^{*}} f_{+}^{D \pi}\left(m_{K^{*}}^{2}\right),
\end{aligned}
$$

where use has been made of

$$
\begin{aligned}
\left\langle V\left(p_{V}\right)|(\bar{s} c)| D\left(p_{D}\right)\right\rangle & =i\left\{\varepsilon_{\mu}\left(m_{D}+m_{V}\right) A_{1}\left(q^{2}\right)-\varepsilon \cdot q \frac{\left(p_{D}+p_{V}\right)_{\mu}}{m_{D}+m_{V}} A_{2}\left(q^{2}\right)\right. \\
& \left.-2 \frac{\varepsilon \cdot q}{q^{2}} q_{\mu} m_{V}\left[A_{3}\left(q^{2}\right)-A_{0}\left(q^{2}\right)\right]\right\}+\frac{2}{m_{D}+m_{V}} \epsilon_{\mu \nu \alpha \beta} \varepsilon^{\nu} p_{D}^{\alpha} p_{V}^{\beta} V\left(q^{2}\right),
\end{aligned}
$$

with $q_{\mu}=\left(p_{D}-p_{V}\right)_{\mu}$ and $A_{3}(0)=A_{0}(0)$. Assuming $\xi_{1} \approx \xi_{2}$ and using the experimental branching ratio $\mathcal{B}\left(D^{+} \rightarrow \bar{K}^{* 0} \pi^{+}\right)=(1.9 \pm 0.7) \%$ [18], and the measured form factors [14]

$$
A_{1}^{D K^{*}}(0)=0.59 \pm 0.04, \quad A_{2}^{D K^{*}}(0)=0.44 \pm 0.09,
$$

and $f_{K^{*}}=0.220$ determined from the decay $\tau \rightarrow K^{*-} \nu_{\tau}$, we get $t^{\text {f }}$

$$
\xi\left(D \rightarrow \bar{K}^{*} \pi\right) \approx-0.22, \quad r\left(D \rightarrow \bar{K}^{*} \pi\right) \approx-1.10
$$

Thus far we have assumed $\xi_{1}=\xi_{2}$ or $r_{1}=r_{2}$. Whether this is true or not can be tested from the decay $D_{s}^{+} \rightarrow \phi \pi^{+}$which occurs solely through the external $W$-emission diagram

$$
A\left(D_{s}^{+} \rightarrow \phi \pi^{+}\right)=\frac{G_{F}}{\sqrt{2}} V_{u d}^{*} V_{c s} a^{\prime}\left(\varepsilon \cdot p_{D_{s}}\right),
$$

with

$$
a^{\prime}=a_{1} f_{\pi}\left[\left(m_{D_{s}}+m_{\phi}\right) A_{1}^{D_{s} \phi}\left(m_{\pi}^{2}\right)-\left(m_{D_{s}}-m_{\phi}\right) A_{2}^{D_{s} \phi}\left(m_{\pi}^{2}\right)\right] .
$$

From $\mathcal{B}\left(D_{s}^{+} \rightarrow \phi \pi^{+}\right)=3.6 \%[21]$ and $\tau\left(D_{s}^{+}\right)=4.50 \times 10^{-13} s$ [18], we find

$$
\left|a^{\prime}\right|=0.25 \mathrm{GeV}^{3} \text {. }
$$

\footnotetext{
${ }^{4}$ For some unknown reasons, the result for $\xi$ determined from the decay $D^{+} \rightarrow \bar{K}^{0} \rho^{+}$is very unsatisfactory: $\xi(D \rightarrow \bar{K} \rho) \sim-1.4$.
} 
If the form factors $A_{1,2}^{D_{s} \phi}$ are taken to be the same as $A_{1,2}^{D K^{*}}$, we then obtain

$$
\xi_{1}\left(D_{s} \rightarrow \phi \pi\right) \approx-0.27, \quad r_{1}\left(D_{s} \rightarrow \phi \pi\right) \approx-1.22
$$

Since $\xi_{1,2}=\left(a_{1,2}-c_{1,2}\right) / c_{2,1}$ and $\left|c_{2}\right|<<\left|c_{1}\right|$, it is clear that the determination of $\xi_{2}$ is far more less uncertain than $\xi_{1}$. Within uncertainties we see that $r_{1}$ and $r_{2}$ are very close. Note that internal $W$ emission is usually suppressed relative to external $W$ emission owing to color mismatch. However, we see from (25) and (34) that internal $W$ emission in $D \rightarrow \phi \pi, D \rightarrow$ $\bar{K} \pi$ dominates over external $W$ emission. Of course, nonfactorizable soft-gluon contribution plays an essential role here.

For the decay $D \rightarrow V V$, its general amplitude reads

$$
A\left(D \rightarrow V_{1} V_{2}\right)=i \frac{G_{F}}{\sqrt{2}} V_{c q_{1}}^{*} V_{q_{2} q_{3}} \varepsilon^{\mu}\left(V_{1}\right) \varepsilon^{\nu}\left(V_{2}\right)\left(\hat{A}_{1} g_{\mu \nu}+\hat{A}_{2} p_{\mu}^{D} p_{\nu}^{D}+i \hat{V} \epsilon_{\mu \nu \alpha \beta} p_{D}^{\alpha} p_{1}^{\beta}\right) .
$$

The first term is an $S$-wave amplitude, the second is a longitudinal $D$-wave term, and the third is a $P$-wave term. We find for $D^{+} \rightarrow \bar{K}^{* 0} \rho^{+}$decay

$$
\begin{aligned}
\hat{A}_{1} & =a_{1} f_{\rho} m_{\rho}\left(m_{D}+m_{K^{*}}\right) A_{1}^{D K^{*}}\left(m_{\rho}^{2}\right)+a_{2} f_{K^{*}} m_{K^{*}}\left(m_{D}+m_{\rho}\right) A_{1}^{D \rho}\left(m_{K^{*}}^{2}\right), \\
\hat{A}_{2} & =-\frac{2}{m_{D}+m_{K^{*}}} a_{1} f_{\rho} m_{\rho} A_{2}^{D K^{*}}\left(m_{\rho}^{2}\right)-\frac{2}{m_{D}+m_{\rho}} a_{2} f_{K^{*}} m_{K^{*}} A_{2}^{D \rho}\left(m_{K^{*}}^{2}\right), \\
\hat{V} & =-\frac{2}{m_{D}+m_{K^{*}}} a_{1} f_{\rho} m_{\rho} V^{D K^{*}}\left(m_{\rho}^{2}\right)-\frac{2}{m_{D}+m_{\rho}} a_{2} f_{K^{*}} m_{K^{*}} V^{D \rho}\left(m_{K^{*}}^{2}\right),
\end{aligned}
$$

where we have applied Eq.(29). Experimentally, a mixture of transverse and longitudinal polarization is found to be consistent with a pure $S$-wave term $[22,18]$ :

$$
\begin{aligned}
& \mathcal{B}\left(D^{+} \rightarrow \bar{K}^{* 0} \pi^{+} ; S \text { - wave }\right)=\left(4.1_{-1.2}^{+1.5}\right) \% \\
& \mathcal{B}\left(D^{+} \rightarrow \bar{K}^{* 0} \pi^{+} ; P \text { - wave }\right)<5 \times 10^{-3}, \\
& \mathcal{B}\left(D^{+} \rightarrow \bar{K}^{* 0} \pi^{+} ; D \text { - wave longitudinal }\right)<7 \times 10^{-3} .
\end{aligned}
$$

It is convenient to write the Lorentz invariant amplitude in terms of three helicity amplitudes:

$$
\begin{aligned}
H_{00} & =\frac{1}{m_{K^{*}} m_{\rho}}\left[\frac{1}{2}\left(m_{D}^{2}-m_{K^{*}}^{2}-m_{\rho}^{2}\right) \hat{A}_{1}+m_{D}^{2} p_{c}^{2} \hat{A}_{2}\right], \\
H_{++} & =\hat{A}_{1}+m_{D} p_{c} \hat{V} \\
H_{--} & =\hat{A}_{1}-m_{D} p_{c} \hat{V}
\end{aligned}
$$

where $p_{c}$ is the c.m. momentum. The decay rate is then given by

$$
\Gamma\left(D^{+} \rightarrow \bar{K}^{* 0} \rho^{+}\right)=\frac{p_{c}}{8 \pi m_{D}^{2}}\left(\left|H_{00}\right|^{2}+\left|H_{++}\right|^{2}+\left|H_{--}\right|^{2}\right) .
$$


Assuming $r_{1}=r_{2}$ as before, $A_{1}^{D \rho}(0) / A_{1}^{D K^{*}}(0)=0.89$ [23], and a monopole behavior for form factors $\left[m_{*}=2.53 \mathrm{GeV}\right.$ for $(\bar{s} c)$ current and $2.42 \mathrm{GeV}$ for $(\bar{u} c)$ current], we obtain

$$
\xi\left(D \rightarrow \bar{K}^{*} \rho\right) \approx-0.34, \quad r\left(D \rightarrow \bar{K}^{*} \rho\right) \approx-1.35
$$

The value of $\xi$ is sensitive to the ratio $A_{1}^{D \rho}(0) / A_{1}^{D K^{*}}(0)$ which has not been measured. Nevertheless, it does not affect the general feature that $|r(D \rightarrow V V)| \gtrsim|r(D \rightarrow V P)|$.

To conclude this section, the parameter $r_{2}$, which measures the nonfactorizable contribution from color octet currents, is found to be of order $\sim-2 / 3,-(0.9 \sim 1.1),-(1.2 \sim 1.3)$ respectively for $D \rightarrow P P, V P, V V$ decays, in accordance with the expectation that softgluon effects become stronger when the final-state particles become less energetic. The parameters $a_{1}$ and especially $a_{2}$ are thus not universal; they are channel or class dependent.

3. Bottom Meson Decays In the exclusive two-body nonleptonic weak decays of bottom mesons, the parameter $a_{1}$ can be estimated directly from neutral $B$ decays, e.g. $\bar{B}^{0} \rightarrow$ $D^{+} \pi^{-}, D^{+} \rho^{-}$. This comes from the fact that, contrary to the charmed meson case, both $W$ exchange and final-state interactions are presumably negligible as the decay particles are very energetic (for a recent study of final-state interactions in $B$ decays, see Ref.[24]). As for the parameter $a_{2}, \bar{B} \rightarrow \psi \bar{K}^{(*)}$ are thus far the only color-suppressed modes which have been measured experimentally. Hence, a direct measurement of $a_{2}$ is available only in these decay modes. A fit to the CLEO II data of $B^{-} \rightarrow \psi K^{-(*)}, \bar{B}^{0} \rightarrow \psi \bar{K}^{0(*)}$ yields [8] (see also Ref.[25])

$$
\left|a_{2}(\bar{B} \rightarrow \psi \bar{K})\right|=0.26 \pm 0.02 \text {. }
$$

Since the Wilson coefficients at the renormalization scale $\mu \sim m_{b}$ are given by

$$
c_{1}\left(m_{b}\right) \sim 1.11, \quad c_{2}\left(m_{b}\right) \sim-0.26
$$

it follows two possibilities: either $a_{2}<0$ and

$$
\xi_{2}(\bar{B} \rightarrow \psi \bar{K}) \approx 0, \quad r_{2}(\bar{B} \rightarrow \psi \bar{K}) \approx-\frac{2}{3}
$$

which is precisely what expected from the leading $1 / N_{c}$ expansion, or $a_{2}>0$ and

$$
\xi_{2}(\bar{B} \rightarrow \psi \bar{K}) \approx 0.47, \quad r_{2}(\bar{B} \rightarrow \psi \bar{K}) \approx 0.27
$$


Unfortunately, what is the sign of $a_{2}$ for $\bar{B} \rightarrow \psi \bar{K}^{(*)}$ decays is still very confusing. On the one hand, a recent calculation based on QCD sum rule indicates a destructive interference between the nonfactorizable soft-gluon term and the subleading $1 / N_{c}$ factorizable term [13]. This in turn implies a negative $a_{2}$. On the other hand, the ratio $a_{2} / a_{1}$ is found to be positive in $B^{-} \rightarrow D \pi, D \rho, D^{*} \pi, D^{*} \rho$ decays [8]. 向

In the following, we will argue that $a_{2}$ appears to be channel dependent: Its sign is opposite in $\bar{B} \rightarrow \psi \bar{K}^{(*)}$ and $\bar{B} \rightarrow D^{(*)} \pi(\rho)$ decays. Following the argument presented in the last section, it is expected that

$$
\left|r_{2}(\bar{B} \rightarrow \psi \bar{K})\right|>\left|r_{2}(\bar{B} \rightarrow D \pi)\right|
$$

A fit of the theoretical calculation [26]

$$
R_{1}=\frac{\mathcal{B}\left(B^{-} \rightarrow D^{0} \pi^{-}\right)}{\mathcal{B}\left(\bar{B}^{0} \rightarrow D^{+} \pi^{-}\right)}=\left(1+1.23 \frac{a_{2}}{a_{1}}\right)^{2}
$$

to the measured value $R_{1}=1.89 \pm 0.26 \pm 0.32[8]$ gives [0

$$
\frac{a_{2}}{a_{1}}(\bar{B} \rightarrow D \pi) \approx 0.30 \text {. }
$$

When combining with $a_{1} \approx 1.05$ determined from $\bar{B}^{0} \rightarrow D^{+} \pi^{-}$, this leads to 0

$$
a_{2}(\bar{B} \rightarrow D \pi) \approx 0.32, \quad \xi_{2}(\bar{B} \rightarrow D \pi) \approx 0.52, \quad r_{2}(\bar{B} \rightarrow D \pi) \approx 0.36
$$

Evidently, the relation (46) can be satisfied only if $a_{2}(\bar{B} \rightarrow \psi \bar{K})$ is negative:

$$
a_{2}(\bar{B} \rightarrow \psi \bar{K})=-(0.26 \pm 0.02)
$$

This argument that the nonfactorizable term and the subleading $1 / N_{c}$ factorizable term in $\bar{B} \rightarrow \psi \bar{K}$ decay are in opposite signs is also in accordance with a recent QCD sum rule calculation [13].

\footnotetext{
${ }^{5}$ The ratio $a_{2} / a_{1}=0.23 \pm 0.11$ obtained in Ref.[8] actually comes from (i) the determination of $\left|a_{1}\right|=$ $1.15 \pm 0.11$ from $\bar{B}^{0} \rightarrow D^{(*)} \pi(\rho)$ decays, (ii) $\left|a_{2}\right|=0.26 \pm 0.02$ from $\bar{B} \rightarrow \psi \bar{K}^{(*)}$ decays, and (iii) the constructive interference in $B^{-} \rightarrow D^{(*)} \pi(\rho)$ decays. A priori there is no reason to expect that $\mid a_{2}(\bar{B} \rightarrow$ $\left.D^{(*)} \pi(\rho)\right)|=| a_{2}\left(\bar{B} \rightarrow \psi \bar{K}^{(*)}\right) \mid$ as the c.m. momentum in $\bar{B} \rightarrow \psi \bar{K}$ is $1683 \mathrm{MeV}$, while it is $2307 \mathrm{MeV}$ in $\bar{B} \rightarrow D \pi$ decay.

${ }^{6} \mathrm{~A}$ direct fit of $a_{2} / a_{1}$ to the other ratios $R_{2}, R_{3}, R_{4}$ (for a definition, see Ref.[26]) also gives a value substantially larger than 0.23 (see Eqs.(27-30) of Ref.[8]).

${ }^{7}$ Unlike the charmed meson decay, $r_{2}$ here is supposed not to vary significantly from $\bar{B} \rightarrow D \pi$ to $\bar{B} \rightarrow D^{*} \rho$ decays.
} 
The most striking feature observed by CLEO [8] is that the parameter $r_{2}\left(\bar{B} \rightarrow D^{(*)} \pi(\rho)\right)$ is positive, recalling that the analogous quantity is always negative in charm decay. If our above argument is correct, we have to understand why the sign of $r_{2}$ changes dramatically from $\bar{B} \rightarrow \psi \bar{K}$ to $\bar{B} \rightarrow D^{(*)} \pi(\rho)$ decays. This should be checked by lattice and QCD sum rule calculations. We see in charmed meson decay that the parameters $r_{1}$ and $r_{2}$ are empirically close. Is this still true in bottom decay? The QCD sum rule calculation in Ref.[12] indicates $r_{1}\left(\bar{B}^{0} \rightarrow D^{+} \pi^{-}\right) \approx-1$ with a sign opposite to $r_{2}\left(B^{-} \rightarrow D \pi\right)$. However, a direct fit to the data of $\bar{B}^{0} \rightarrow D^{(*)} \pi(\rho)$ (except for $\bar{B}^{0} \rightarrow D^{+} \rho^{-}$) gives a value of $a_{1}$ of order $1.01 \sim 1.05$ (see Ref.[24] and Table XX of Ref.[8]) with the neglect of $W$ exchange and final-state interactions. It thus appears that the sign of $r_{1}\left(\bar{B}^{0} \rightarrow D^{+} \pi^{-}\right)$could be the same as that of $r_{2}\left(B^{-} \rightarrow D \pi\right)$. This issue should be clarified in the future.

4. Conclusions We have considered the nonfactorizable contributions to exclusive twobody nonleptonic weak decays of charmed and bottom mesons arising from color octet currents. From the data we have determined the parameter $r_{2}$, which measures the soft gluon effects, to be $\sim-0.67,-(0.9 \sim 0.11),-(1.2 \sim 1.3), \sim 0.36$ respectively for $D \rightarrow$ $\bar{K} \pi, \bar{K}^{*} \pi, \bar{K}^{*} \rho$, and $\bar{B} \rightarrow D \pi$ decays. Therefore, the soft-gluon effects become more important when final-state particles become less energetic, as expected. In the $1 / N_{c}$ approach, the subleading contribution is characterized by the parameters $\xi_{i}=\left(1 / N_{c}+r_{i} / 2\right)(i=1,2)$. It is evident that the coefficients $a_{1}\left(=c_{1}+\xi_{1} c_{2}\right)$ and especially $a_{2}\left(=c_{2}+\xi_{2} c_{1}\right)$ are not universal; they are decay mode or class dependent.

The very striking feature observed by CLEO that $r_{2}$ is positive for $\bar{B} \rightarrow D \pi$ decays, whereas it is always negative in charmed meson decays, needs to be checked by lattice and QCD sum rule calculations. We have argued that for $\bar{B} \rightarrow \psi \bar{K}^{(*)}$ decays, the nonfactorizable term contributes destructively with the $1 / N_{c}$ factorizable term so that the sign of $a_{2}$ is negative. As a consequence, the leading $1 / N_{c}$ expansion turns out working well for $\bar{B} \rightarrow$ $\psi \bar{K}^{(*)}$, though it fails for $\bar{B} \rightarrow D^{(*)} \pi(\rho)$ decays.

\footnotetext{
${ }^{8}$ This number is taken from Ref.[27], which is smaller than the original estimate $r_{1} \sim-1.5$ given in Ref.[12].
} 


\section{ACKNOWLEDGMENT}

This work was supported in part by the National Science Council of ROC under Contract No. NSC83-0208-M-001-014.

\section{References}

1. D. Gross, talk presented at the XVI International Symposium on Lepton-Photon Interactions, Ithaca, 10-15 August 1993.

2. H. Georgi, Annu. Rev. Nucl. Part. Sci. 43, 209 (1993).

3. R. Dashen and A.V. Manohar, Phys. Lett. B315, 425, 438 (1993); R. Dashen, E. Jenkins, and A.V. Manohar, Phys. Rev. D49, 4713 (1994).

4. A.J. Buras, J.-M. Gérard, and R. Rückl, Nucl. Phys. B268, 16 (1986).

5. H.Y. Cheng, Int. J. Mod. Phys. A4, 495 (1989).

6. M. Fukugita, T. Inami, N. Sakai, and S. Yazaki, Phys. Lett. 72B, 237 (1977); D. Tadić and J. Trampetić, Phys. Lett. 114B, 179 (1982).

7. M. Bauer, B. Stech, and M. Wirbel, Z. Phys. C34, 103 (1987).

8. CLEO Collaboration, M.S. Alam et al., CLNS 84-1270 (1994).

9. H.Y. Cheng, Z. Phys. C32, 237 (1986).

10. N. Deshpande, M. Gronau, and D. Sutherland, Phys. Lett. 90B, 431 (1980); M. Gronau and D. Sutherland, Nucl. Phys. B183, 367 (1981).

11. B. Blok and M. Shifman, Sov. J. Nucl. Phys. 45, 35, 301, 522 (1987).

12. B. Blok and M. Shifman, Nucl. Phys. B389, 534 (1993).

13. A. Khodjamirian and R. Rückl, MPI-PhT/94-26 (1994). 
14. M. Witherell, talk presented at the XVI International Symposium on Lepton-Photon Interactions, Ithaca, 10-15 August 1993.

15. Mark III Collaboration, J. Adler et al., Phys. Rev. Lett. 62, 1821 (1989).

16. CLEO Collaboration, M.S. Alam et al., Phys. Rev. Lett. 71, 1311 (1993).

17. CLEO Collaboration, M. Selen et al., Phys. Rev. Lett. 71, 1973 (1993).

18. Particle Data Group, Phys. Rev. D45, S1 (1992).

19. L.L. Chau and H.Y. Cheng, ITP-SB-93-49, to appear in Phys. Lett. B.

20. L.L. Chau and H.Y. Cheng, Phys. Rev. Lett. 56, 1655 (1986); Phys. Rev. D36, 137 (1987); Phys. Lett. B222, 285 (1989).

21. F. Muheim and S. Stone, Phys. Rev. D49, 3767 (1994).

22. Mark III Collaboration, D. Coffman et al., Phys. Rev. D45, 2196 (1992).

23. M. Wirbel, B. Stech, and M. Bauer, Z. Phys. C29, 637 (1985).

24. H. Yamamoto, HUTP-94/A006 (1994).

25. A. Deandrea, N. Di Bartolomeo, R. Gatto, and G. Nardulli, Phys. Lett. B318, 549 (1993); K. Honscheid, K.R. Schubert, and R. Waldi, OHSTPY-HEP-E-93-017 (1993); S. Resag and M. Beyer, BONN-TK-93-18 (1993); M. Gourdin, A.N. Kamal, Y.Y. Keun, and X.Y. Pham, PAR/LPTHE/94-22 (1994).

26. M. Neubert, V. Riekert, Q.P. Xu, and B. Stech, in Heavy Flavors, edited by A.J. Buras and H. Lindner (World Scientific, Singapore, 1992).

27. I. Bigi, B. Blok, M. Shifman, N. Uraltsev, and A. Vainshtein, CERN-TH.7132/94 (1994). 